Nota científica

\title{
NUEVA LOCALIDAD PARA EL TREPATRONCOS GIGANTE (XIPHOCOLAPTES PROMEROPIRHYNCHUS) EN EL ESTADO DE GUERRERO, MÉXICO
}

\begin{abstract}
In February of 2006 an individual of the Strong-billed Woodcreeper was sighted near the locality of Juvinani, municipality of Metlatonoc, in the Mexican state of Guerrero. This is the first sighting reported for this species in the region and increases its distribution in $135 \mathrm{~km}$ within the state of Guerrero.
\end{abstract}

Las aves conocidas como trepatroncos pertenecen a la familia Furnariidae y son de distribución neotropical (Banks et al. 2006, Raikow 1994). El trepatroncos gigante (Xiphocolaptes promeropirhynchus) es la única especie de este género en México y es el de mayor tamaño en el país. De acuerdo con Howell \& Webb (1995), en México se distribuye en la vertiente del Atlántico, desde el SE de San Luis Potosí hacia Veracruz y en la vertiente del Pacífico incluye a Guerrero, Oaxaca y Chiapas. En Guerrero esta especie es registrada sólo en la región de Omiltemi. (Griscom 1937, Howell \& Webb 1995, Martin del Campo 1948, Navarro-Sigüenza 1998, información no publicada, Nelson 1903).

Con la finalidad de corroborar la información de su distribución, se consultaron las bases de datos de museos y colecciones disponibles a través de los portales electrónicos: Global Biodiversity Information Facility (GBIF; www.gbif.net, julio del 2006), Ornis (http://olla.berkeley.edu/ornisnet, julio de 2006) y la Red Mundial de Información sobre Biodiversidad (REMIB; http://www.conabio.gob.mx/ remib/doctos/remib_esp.html, julio de 2006).

Dentro del estado de Guerrero Navarro-Sigüenza (1998, datos no publicados) menciona la presencia de ocho especies de trepatroncos: Sittasomus griseicapillus, mide de 15 a $16.5 \mathrm{~cm}$ y tiene un pico pequeño y delgado. Dendrocolaptes certhia tiene una longitud entre los 22.5 a $28 \mathrm{~cm}$ y el cuerpo es café con barrado obscuro, aun en la garganta. Xiphorhynchus flavigaster mide de 21.5 a $26.5 \mathrm{~cm}$, es de amplia distribución dentro del estado, su pico es blanco y la espalda moteada en claro. Xiphorhynchus erythropigius $(23-24 \mathrm{~cm})$ posee sobre todo el cuerpo, principalmente en la espalda y garganta, manchas blancas. De las especies del género Lepidocolaptes, L. leucogaster $(21 \mathrm{a} 23 \mathrm{~cm})$, se caracteriza por el blanco de su parte ventral, L. soleyetti posee una coloración clara y es más pequeño $(20 \mathrm{~cm})$ y L. affinis $(21 \mathrm{~cm})$ es de tamaño reducido y con manchas de color ante en espalda y vientre, y garganta clara.

Durante febrero de 2006 en un viaje para explorar la biodiversidad de Guerrero, se observó a un individuo de Xiphocolaptes promeropirhynchus sobre el tronco de un Pinus sp, en una ladera cerca de Yuvinavi (17²'57.9” LN y 98²4'9.62” LO), en el municipio de Metlatónoc, Gro. (Fig. 1). Este registro de diez minutos, fue obtenido 
a las 09:29 horas cuando estaba forrajeando con movimientos de ascensión vertical y girando alrededor del tronco.

Las características que permiten aseverar que el individuo observado corresponde a esta especie son: el plumaje aparentemente liso del pecho contra el café barrado señalado para Dendrocolaptes certhia, la garganta blanca, que no posee D. certhia, y lo masivo del pico. En comparación con las otras especies, Xiphocolaptes promeropirhynchus es un trepatroncos de mayor tamaño que hace fácilmente descartables a las restantes especies. El hábitat donde se registró corresponde a bosque de pino-encino y amplía la distribución de la especie en $135 \mathrm{~km}$ dentro del estado. En Guerrero se reconoce la subespecie Xiphocolaptes promeropirhynchus omiltemensis, en las inmediaciones de Omiltemi, y los registros pertenecen precisamente al trepatroncos gigante de Omiltemi, subespecie considerada en peligro de extinción por la legislación ambiental mexicana (NOM-059-ECOL-2001).
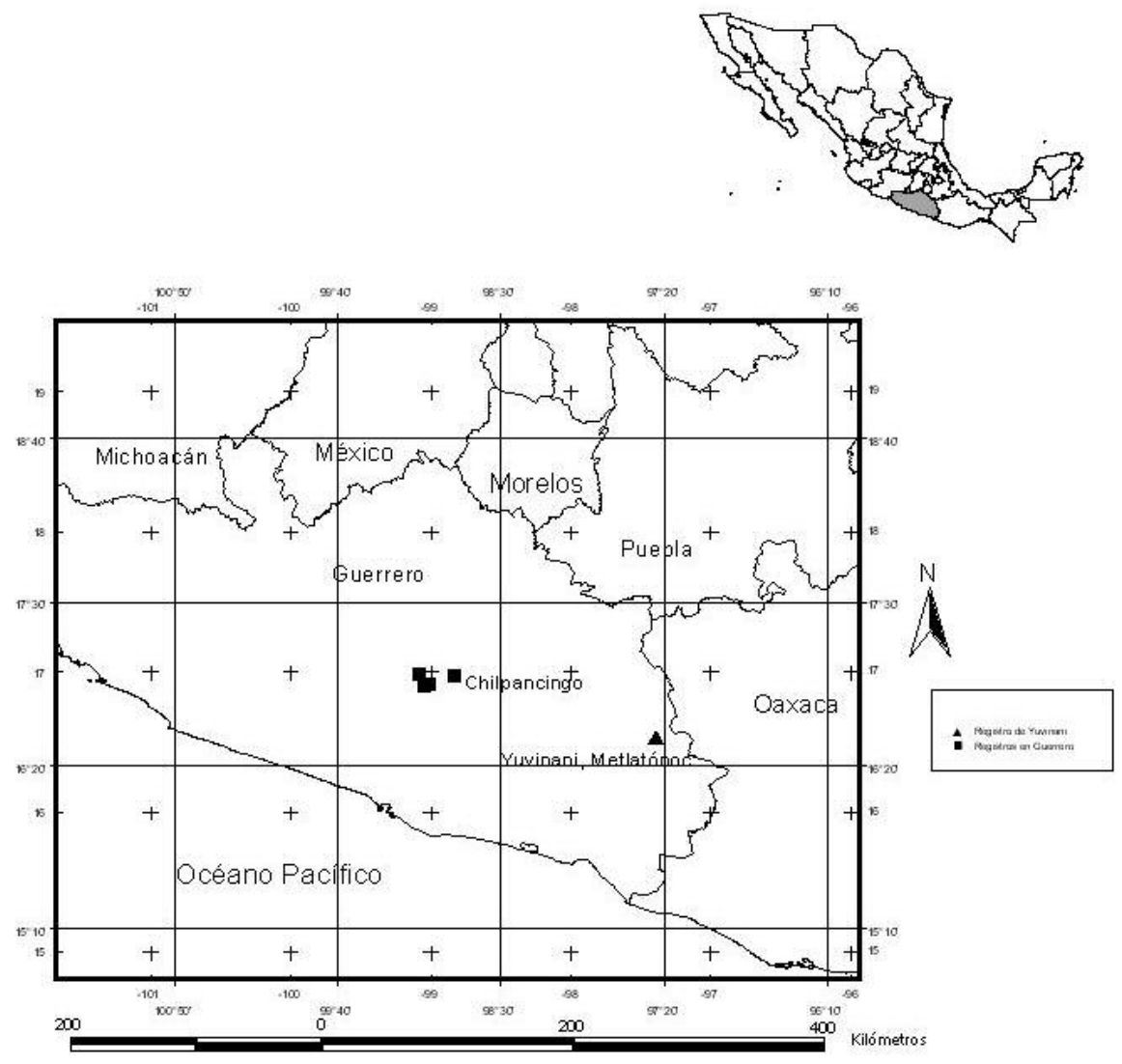

Figura 1. Registros actuales de para Guerrero (cuadrados) y Oaxaca (círculos). La zona del nuevo registro está marcada con un triángulo. 
Agradecimientos: A los miembros del cuerpo académico Diagnóstico de la Diversidad, de la UAEM, Rubén Castro Franco que revisó el documento final, Jorge A. Viana Lases por la información sobre la flora y Hugo Zagal Maldonado por la información cartográfica. A Fernando Urbina Torres y César Jiménez Piedragil del Laboratorio de Ornitología de la UAEM por sus comentarios respecto a la distribución.

\section{LITERATURA CITADA}

Banks, R. C., C. Cicero, J: L: Dunn, A. W. Kratter, P. C. Rasmussen, J.V. Remsen, J. D. Rising \& D. F. Stots. 2006. Forty-seventh supplement to the American Ornithologists' Union check-list of North American birds. The Auk 123(3): 926-936

Griscom, L. 1937. A collection of birds from Omilteme, Guerrero. The Auk 54(2): 192-199

Howell, S.N.G. \& S. Webb. 1995. A guide to the birds of Mexico and Northern Central America. Oxford University Press, Oxford New York.

Martín Del Campo, R. 1948. Contribución para el conocimiento de la fauna ornitológica del estado de Guerrero. Anales del Instituto de Biología, Universidad Nacional Autónoma de México 19(1): 241266.

Nelson, E. W. 1903. Descriptions of new birds from southern Mexico. Proceedings of the Biological Society of Washington. 16: 151-160.

Raikow, R. J. 1994. A phylogeny of the Woodcreepers (Dendrocolaptinae). The Auk 111(1): 104-114.

\section{Aquiles Argote Cortés}

Laboratorio de Ornitología, Centro de Investigaciones Biológicas, Universidad Autónoma del Estado de Morelos. Av. Universidad 1001, Chamilpa. Cuernavaca, 62209, Morelos, México.

argote@cib.uaem.mx 